\title{
Semiconductor snail lasers
}

\author{
M. J. Strain, ${ }^{1, a)}$ G. Mezősi, ${ }^{1}$ J. Javaloyes, ${ }^{1}$ M. Sorel,,${ }^{1}$ A. Pérez-Serrano, ${ }^{2}$ A. Scirè,${ }^{2}$ \\ S. Balle, ${ }^{3}$ J. Danckaert, ${ }^{4}$ and G. Verschaffelt ${ }^{4}$ \\ ${ }^{1}$ Department of E\&EE, University of Glasgow, Glasgow G12 8LT, United Kingdom \\ ${ }^{2}$ IFISC, UIB-CSIC, Campus UIB, E-07122 Palma de Mallorca, Spain \\ ${ }^{3}$ IMEDEA, C/Miquel Marqués, 21; E-07190 Esporles, Spain \\ ${ }^{4}$ Department of Applied Physics and Photonics, Vrije Universiteit Brussel, Pleinlaan 2, \\ 1050 Brussels, Belgium
}

(Received 26 November 2009; accepted 2 March 2010; published online 23 March 2010)

\begin{abstract}
A modified ring laser geometry is presented to promote stable unidirectional lasing. The effects of directional coupling and facet reflectivities are investigated with respect to quantum efficiency, directionality, and side-mode suppression ratio of the lasing spectra. Simulation and experimental results are presented showing single mode $(>20 \mathrm{~dB}$ side-mode suppression ratio), unidirectional lasing on an InP based multiple quantum well material. () 2010 American Institute of Physics. [doi:10.1063/1.3371721]
\end{abstract}

Semiconductor ring lasers (SRL) are becoming a mature and versatile technology with applications in telecommunications, optical signal processing, and on-chip sensing. Research carried out into SRL devices has demonstrated many of the functions that have been previously presented in Fabry-Pérot (FP) and distributed feedback lasers; such as tunability, ${ }^{1}$ master-slave operation, ${ }^{1}$ and mode-locking. ${ }^{2}$ Additionally, directional bistability has been demonstrated, ${ }^{3}$ with possible future applications in optical memory and delay lines. Further application of SRLs may be made in high power applications, especially where spatially dense arrays of outputs are required, for example, in high resolution printing heads. SRLs do not suffer from the catastrophic optical damage (COD) that occurs in FP lasers where the cleaved facets are employed as feedback elements in the laser cavity. In SRLs, the lasing cavity is formed by the closed ring waveguide with light extracted using waveguide coupler structures, avoiding inclusion of facets in the lasing cavity. In addition, SRLs may be spatially separated on chip, allowing better thermal management, and contact definition for individual addressing, without the considerations that would be necessary for FP devices, such as waveguide bends or etched cavity mirrors. However, in SRLs it is difficult to realize the equivalent to high and low reflectivity facet coatings, which allow increased quantum efficiency and directionally dependent output power. The symmetric nature of the coupling in SRLs can produce bidirectional or (fluctuating) bistable directional lasing of the device. ${ }^{4}$ Bistable unidirectionality relies on minimizing reflections within the laser cavity, which, by coupling energy between counter-propagating modes leads to bidirectional emission. So, the necessity for high output coupling for increased quantum efficiency of SRLs also results in increased feedback into the laser cavity and hence, bidirectional lasing. In this paper, an alternative "snail" ring laser geometry is presented as a means to produce high efficiency, stable unidirectional lasing.

Figure 1 shows a schematic of the snail laser device. The structure is similar to that of conventional SRLs but the cavity is now defined through the crossed ports of the evanescent coupler. The waveguide inside this ring has an etched

${ }^{a)}$ Electronic mail: mstrain@elec.gla.ac.uk. facet, and the one outside is tilted and tapered. This laser geometry is proposed with the objectives of achieving highly directional output with high slope efficiency. The unbalance of the reflectivity of the two facets couples a large fraction of the clockwise $(\mathrm{CW})$ mode (as defined by the schematic in the inset of Fig. 2) into the counter-clockwise (CCW) mode, so creating unidirectional lasing in this direction. The use of the crossed ports for defining the laser cavity allows us to achieve high slope efficiency with conventional evanescent couplers. In a SRL geometry this would entail producing a directional coupler (DC) with a high coupling fraction, which requires a compromise between small gaps separating the copropagating waveguides (requiring highly optimized pattern definition and etching) and coupler lengths (leading to device lengths in the order of hundreds of microns). By using the snail geometry this problem is inverted, and the requirement for low coupling DCs, improves the fabrication tolerances significantly, allowing the production of short, widely spaced copropagating waveguides.

The theoretical analysis of the snail laser is based on the traveling-wave model (TWM) developed in Ref. 5, with the boundary conditions appropriate to this geometry, as depicted in the inset of Fig. 2, where $r_{3}\left(r_{2}\right)$ is the tail (output facet) reflectivity, $L_{3}\left(L_{2}\right)$ is the length of the tail (output) waveguide, $L$ is the length of the cavity, and $\delta^{2}$ is the crosscoupling factor of the DC.

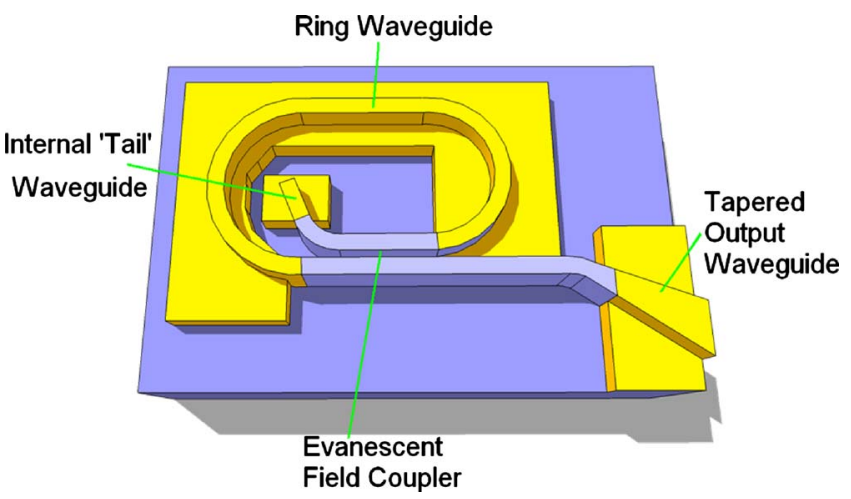

FIG. 1. (Color online) Schematic of a snail laser device. 


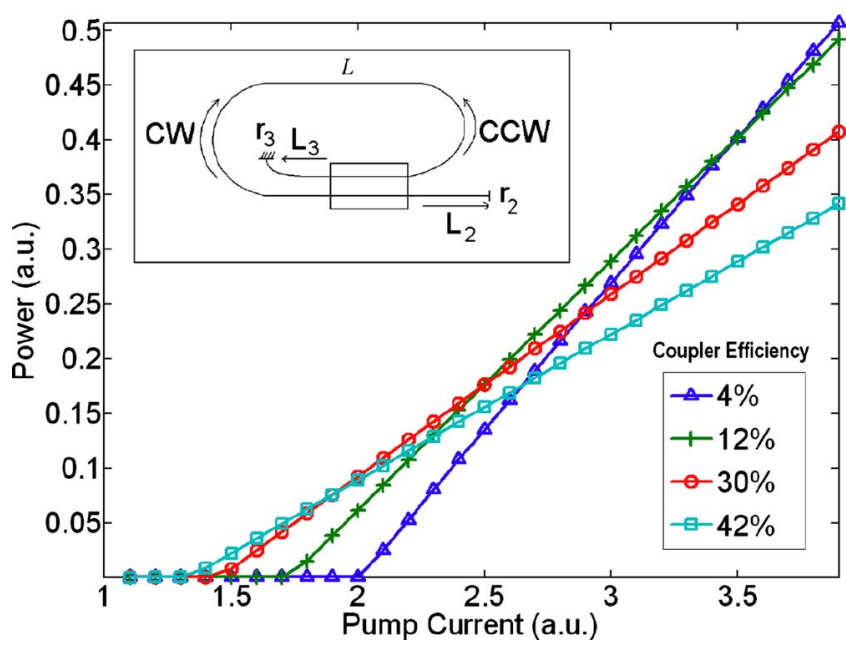

FIG. 2. (Color online) TWM simulation of LI curves outside facet 2 (CCW mode of the ring) for varying $\delta$ in a snail laser device.

The lasing modes of the snail laser can be determined by a transfer-matrix analysis as in Ref. 6. The characteristic equation for the allowed propagation constants $q$ reads

$$
Z^{2}\left(\delta^{2}+Z_{2} Z_{3}\right)+2 i \delta\left(1+Z_{2} Z_{3}\right) Z-1-Z_{2} Z_{3} \delta^{2}=0,
$$

where $Z=e^{i q L}, Z_{2(3)}=r_{2(3)} e^{i \omega n_{2(3)} L_{2(3)} / c}, \omega$ is the optical frequency, and $n_{2(3)}$ is the effective index of the output (tail) waveguide. The characteristic equation has two branches of solutions $q_{m}^{ \pm}(m=0,1,2, \ldots)$, which define the allowed cavity modes. The threshold gain for each mode is given by $\operatorname{Im}\left(q_{m}^{ \pm}\right)$, the imaginary part of $q_{m}^{ \pm}$, so that the two solutions corresponding to the same value $m$ have, for $r_{2} r_{3} \ll \delta^{2}$, a threshold difference given by

$$
\Delta \operatorname{Im}\left(q_{m}^{ \pm}\right)=2 \frac{1-\delta^{2}}{\delta L} \sqrt{r_{3} r_{2}} \cos \left(\omega \frac{n_{3} L_{3}+n_{2} L_{2}}{2 c}\right) .
$$

The effective loss modulation due to the compound-cavity effects in the output waveguides may degrade side-mode suppression ratio (SMSR) in the device. Since the amplitude of the loss modulation increases as $\delta$ decreases, in order to produce a high output laser source with high SMSR, small reflectivity of the tail and output facet is required. Moreover, since the ratio of powers emitted through the output and tail ports is given by

$$
\frac{P_{2}}{P_{3}}=\frac{1-r_{2}^{2}}{1-r_{3}^{2}} \frac{r_{3}}{r_{2}},
$$

the external efficiency can be enhanced by increasing $r_{3}$ and lowering $r_{2}$, as anticipated.

Figure 2 shows the simulated LI curves given the facet reflectivities, and varying $\delta$. In the simulations, the output waveguides have been considered as passive. The TWM shows an inverse relationship between the cross-coupling factor $\delta$ and both the threshold current and slope efficiency.

In all of the designs presented here, the waveguide width is $2 \mu \mathrm{m}$, the output waveguide was tapered up to $8 \mu \mathrm{m}$, and the device bar facet cleaved at $80^{\circ}$ to the waveguide normal in order to minimize backreflections. The average ring radius around the snail cavity is $200 \mu \mathrm{m}$. The length of the output waveguides is $450 \mu \mathrm{m}$ and the tail waveguides are $208 \mu \mathrm{m}$ long. The tail waveguide incorporates a contact that allows control of its effective reflectivity by applying a bias. FDTD

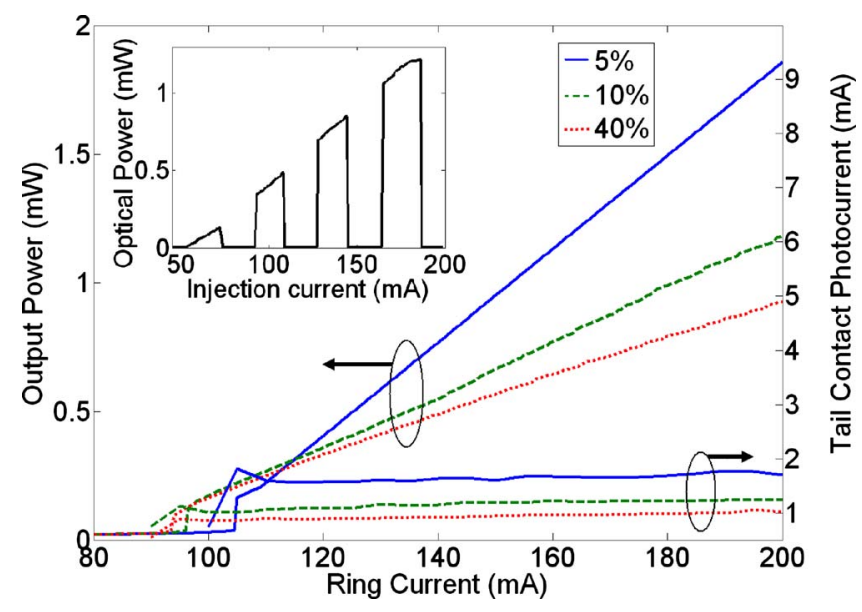

FIG. 3. (Color online) LI curves for snail laser devices with varying crosscoupling factor measured from external facet (CCW mode of the ring), and photocurrent measurement (CW mode of ring) on the tail contact. (Inset: LI curve from a SRL device.)

simulations of the structures gave values of $6.1 \%$ and $0.67 \%$, respectively, for the tail and output facet reflectivities at transparency. Devices with coupler lengths of 56.4, 80.5, and $171 \mu \mathrm{m}$ were produced, giving nominal coupling of $5 \%, 10 \%$, and $40 \%$, respectively. The devices were fabricated on a multiquantum-well (QW) InAlGaAs/InP wafer structure with a gain region consisting of five $6 \mathrm{~nm}$ compressively strained InAlGaAs wells and six $10 \mathrm{~nm}$ slightly tensile strained barriers. The waveguiding core is formed by a $60 \mathrm{~nm}$ graded-index layer on either side of the QW region. Hydrogen silsesquioxane (HSQ) was used as a negative tone electron beam resist for definition of the waveguides. After exposure and development the HSQ pattern was used as a hardmask for the etching of the uppercladding layers using a $\mathrm{CH}_{4} / \mathrm{H}_{2} / \mathrm{O}_{2}$ reactive ion etch.

Experimental LI curves for the lasers were measured by coupling the output from the tapered facet to a broad area $\mathrm{Ge}$ photodetector. Figure 3 shows LI curves for the snail laser devices with the tail contact biased at $0 \mathrm{~V}$. As predicted by the model, the threshold current and slope efficiency of the snail laser devices increase as $\delta$ decreases. The abrupt step in power at the lasing thresholds is due to the fact that the DC sections are unpumped, acting as saturable absorbing sections. Photocurrent measurements of the tail waveguide contact confirmed unidirectional operation. Moreover, the LI curves show no evidence of the directional switching that occurs in SRLs (see inset for a comparison). ${ }^{4}$ Directional switching could be observed only for the $40 \%$ coupled snail laser for reverse bias $<-2 \mathrm{~V}$ on the tail contact, suggesting that as $r_{3} \rightarrow r_{2}$, the laser is no longer forced to operate unidirectionally in the $\mathrm{CCW}$ direction.

Spectra of the snail laser devices under varying injection current, and reverse bias on the tail waveguide contact, were also recorded. Figure 4(a) exhibits the SMSR of the lasers at two times threshold current for varying reverse bias $V_{R}$ on the tail waveguide contact.

For $V_{R}=-3 \mathrm{~V}$ (i.e., low $r_{3}$ ), the SMSR is largest for the device with highest $\delta$, in agreement with the theoretical prediction. For the 5\% coupler device, the SMSR does not vary greatly with $V_{R}$, as expected from the correspondingly large loss modulation amplitude [see Eq. (2)]. Instead, the SMSR of the $10 \%$ and $40 \%$ coupler devices strongly varies with 


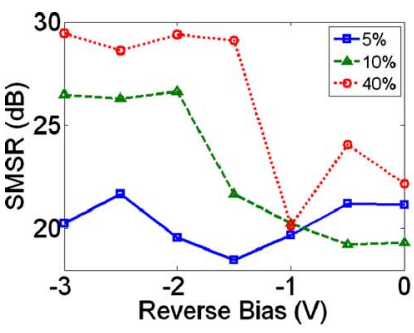

(a)

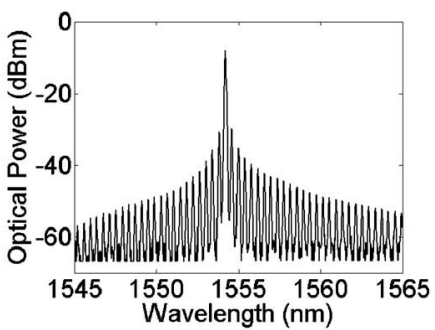

(b)

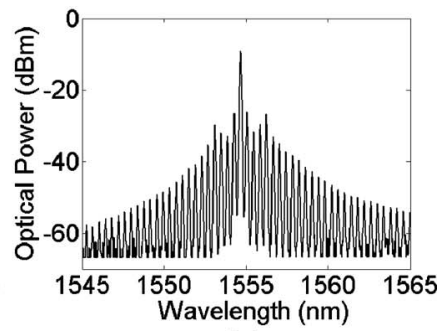

(c)

FIG. 4. (Color online) (a) SMSR vs reverse bias on internal waveguide for snail lasers with varying coupling factor, [(b) and (c)] spectra for snail laser with $10 \%$ coupler and $V_{R}=-3 \mathrm{~V}$ and $V_{R}=0 \mathrm{~V}$, respectively.

$V_{R}$, abruptly falling by $\sim 8 \mathrm{~dB}$ above $V_{R}=-2 \mathrm{~V}$ and $V_{R}=-1.5 \mathrm{~V}$. This indicates the crossover to a regime of stronger coupled cavity effects which manifests as an enhancement of secondary modes in the optical spectra. The coupled cavity effect can be clearly identified in Fig. 4(c) as opposed to the single mode spectra of Fig. 4(b).

Finally, in order to demonstrate potential of creating dense arrays of these devices on a single chip, a set of four devices were fabricated as shown in Fig. 5, with an output waveguide pitch of $32 \mu \mathrm{m}$. A near field image is also shown, exhibiting the four distinct output spots of the devices, each of which was individually addressable.

In conclusion, a modification of a ring laser geometry has been presented in order to produce a high efficiency,

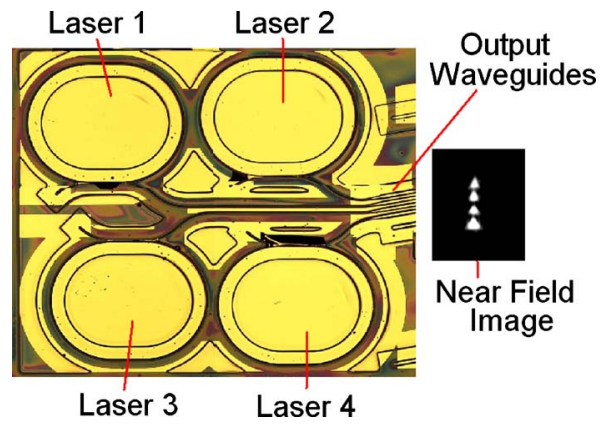

FIG. 5. (Color online) Micrograph of snail laser array, with near field image of emission from four output waveguides. unidirectional laser source without suffering from the COD problems associated with FP lasers. SMSRs of over $20 \mathrm{~dB}$ were exhibited for all of the devices fabricated, with increased quantum efficiency being exhibited with reducing coupling factor. Increasing the reflectivity from the internal waveguide promotes unidirectionality, but then coupled cavity effects emerge, reducing the SMSR, especially for devices with low $\delta$. The simulations carried out using the TWM, for the threshold, slope efficiency and SMSR behavior of the devices are in good agreement with the experimental results. An array of four $32 \mu \mathrm{m}$ output pitched, individually addressable snail lasers was also presented.

We acknowledge financial support from the EU Grant No. FP6-2005-IST-5 through project IOLOS (034743) and partial support of S. Balle through project TEC2006-13887C05-03, and of J. Danckaert and G. Verschaffelt from IAP $\mathrm{VI} / 10$ and FWO. The authors would like to thank D. Yanson, M. Silver, the staff of the JWNC and B. Ward.

${ }^{1}$ S. Furst, S. Yu, and M. Sorel, IEEE Photonics Technol. Lett. 20, 1926 (2008).

${ }^{2}$ Y. Barbarin, E. A. J. M. Bente, M. J. R. Heck, Y. S. Oei, R. Notzel, and M. K. Smit, Opt. Express 14, 9716 (2006).

${ }^{3}$ M. Hill, Nature (London) 432, 206 (2004).

${ }^{4}$ M. Sorel, G. Giuliani, A. Scire, R. Miglierina, S. Donati, and P. Laybourn, IEEE J. Quantum Electron. 39, 1187 (2003).

${ }^{5}$ J. Javaloyes and S. Balle, IEEE J. Quantum Electron. 45, 431 (2009).

${ }^{6}$ S. Fürst, A. Pérez-Serrano, A. Scirè, M. Sorel and S. Balle, Appl. Phys. Lett. 93, 251109 (2008). 